\title{
MAC Layer Communication Protocol design using Stochastic Network Calculusfor Underwater Agriculture Farming
}

\author{
Saravanan M' ${ }^{1}$, Rajeev Sukumaran ${ }^{2}$, MR Christhuraj ${ }^{3}$, Manikandan TT ${ }^{4}$ \\ ${ }^{1}$ Research scholar, ${ }^{124}$ Computer Science and Engineering, SRM Institute of Science and Engineering, \\ Potheri, Tamilnadu. \\ ${ }^{3}$ Information Technology, M.Kumarasamy College of Engineering, Karur, Tamilnadu. \\ ${ }^{1}$ saravanan.be88@gmail.com
}

Article History: Received: 11 January 2021; Accepted: 27 February 2021; Published online: 5 April 2021

\begin{abstract}
The hazardous in seawater network, channel utilization, and MAC layer protocol design induces the research challenges and opportunities of underwater acoustic communications, particularly in terms of throughput and transmission delay. In this research work, we propose the delay-tolerant MAC protocol with collision avoidance. Under_Water Medium Access control and Collision Avoidance -Wireless protocol (UWMACA-W) proposed for underwater Agriculture Farming. This research work also has compared the performance of the UWMACA-Wireless protocol with and without SNC. The growth of the plants inside the bubble can be exchanged to the base station by using the UWMACA-wireless protocol and also increases interface efficiency by taking account of the underwater acoustic channel's long delay time, as well as fixing the issues related to uncovered terminal issues. UWMACA-W method has higher performance than MACAWireless protocol, according to simulation testing on Riverbed modeler.
\end{abstract}

Keywords: Underwater Acoustic Wireless Communication, Delay, Backlog, Stochastic Network Calculus, Underwater agriculture forming.

\section{Introduction}

Submerged structures grasp different applications in obstruction findings in the direction of the ocean oil industry, and other business applications [1].Scientists are searching for new methods and strategies for agriculture and farming due to the world's population increase.An alternative agricultural method that involves growing terrestrial crops in the sea.Underwater farming is a novel and forward-thinking method of agriculture. Diagonal cultivation and hydroponic systems are two more modern farming techniques. Underwater farming is a novel and forward-thinking method of agriculture.Many of the difficulties associated with conventional farming disappear underwater, while plants' essential needs are fulfilled. The sunshine that each plant requires enters the biospheres while being protected from inclement weather such as hail and parasitic attacks. The underwater farming setup and plant growth are illustrated in Fig1. it shows the bubble setup for underwater forming and Fig 1.b. shows the growth of the crops inside the bubble setup.

The growth rate, temperature, and humidity level ratio measurement is a necessary activity for underwater agriculture.Physically monitoring these factors in every moment is a difficult one as a human being.The underwater wireless acoustic communication needs to develop for information exchange between the nodes which are deployed inside the bubble to the base. Submergence of seawater provides a steady temperature while preventing exposure to harsh weather conditions on soil, unlike underground hydroponic systems and greenhouses, which rely on multiple heating and cooling systems to control the temperature. The conversation is carried out in an aquatic channel of communication, it is not equivalent to the traditional terrestrial communication methods [2]. It's one of the most difficult situations the researchers have ever faced to utilize the acoustic communication channel.

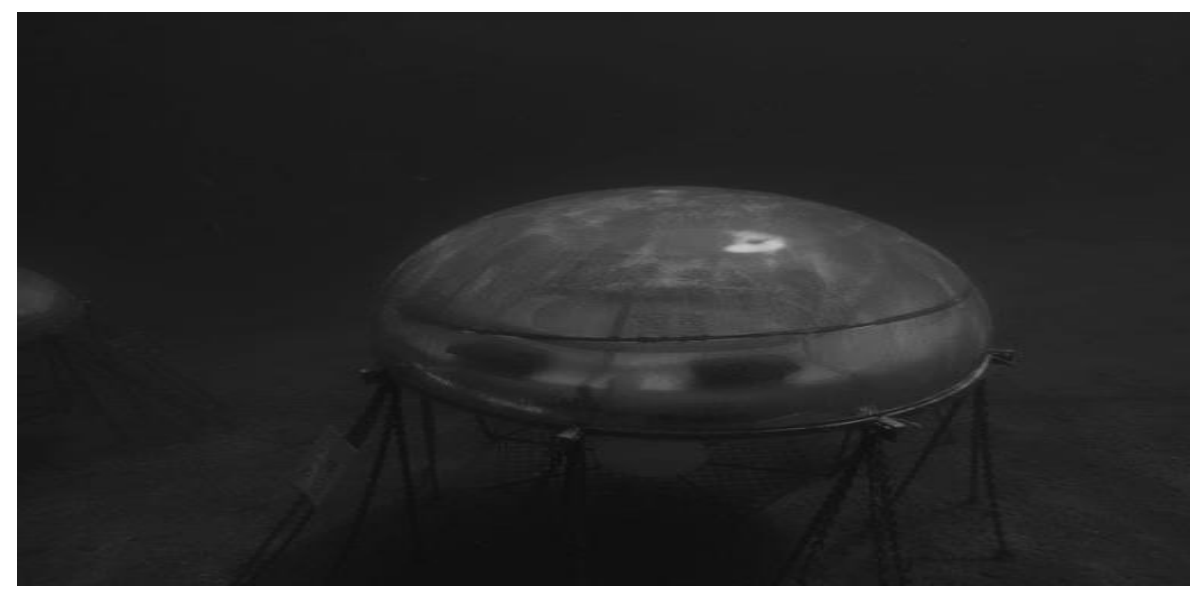

Fig 1.a. Realistic Bubbles setup for the underwater form 


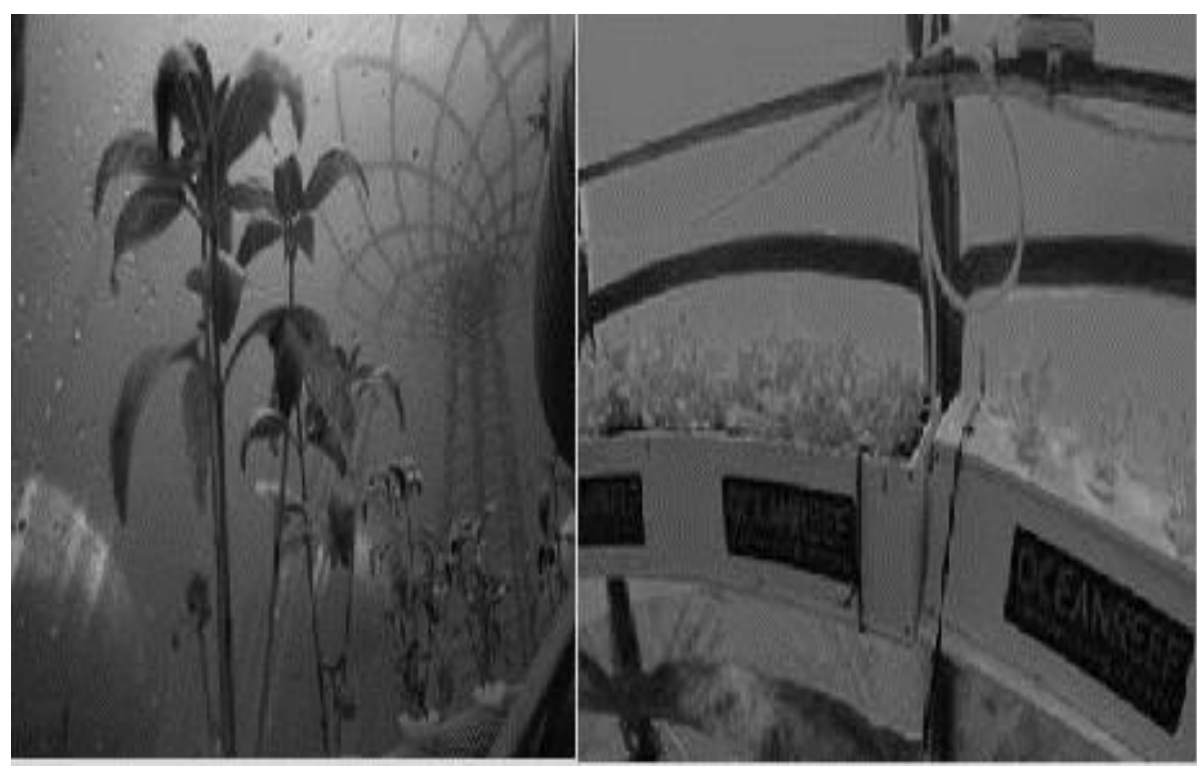

Fig 1.b. plants growth inside the underwater form

An acoustic channel communication between the nods and the base station is affected by the variety of acoustic channels. The short distance communication can be received by the transceivers. Since the growth of the underwater system is not automated [3]. The MAC protocols need to test with underwater forming. This paper deals with wireless underwater MAC protocol to measure and indicates the growth rate of the crops inside the bubbles to the outside station. Wireless underwater MAC protocols dealing with acoustic channel allocation and collision issues[4]. The lowered acoustic channel is difficult to receive the data due to crippling, multipath concealing, and time comparing ascribes [5]. The acoustic spread is often smaller than the radio channel, creating uncertainty [6] [15]. The transmission speed of acoustic signals in the seabed is around $1500 \mathrm{~m} / \mathrm{s}$, which is below the range of radio propagating waves. Furthermore, because of the restricted space available in submerged channels, Frequency Division Multiple Access (FDMA) is not ideal for submerged communications [7] [18]. Collision avoidance protocols are expected to minimize re-transmissions and save resources that are battery-powered. These unfavorable properties make it difficult to design effective and efficient communication protocols [8].

In recent years, several MAC protocols have been invented and updatedatthe simulation level. Since there is no proper collision [11] [12] [13] avoidance method is encounter stochastically in the ALOHA or MACA (Collision Avoidance). A device can automatically relay a payload if it has something to transmit. Whereas the network's loading is heavy, the channel's performance degrades exponentially owing to the unavailability of any collision avoidance system [9] [14].To minimize packet losses, CSMA allows nodes to listen/sense the channel, and it solves the issue of unseen and exposed terminals. Later Wireless-medium access collision avoidance interprocess communication protocols [10] are presented to overcome the CSMA issue, but they fail spectacularly when implemented underwater.At this moment, propose a UWMACA (Underwater Wireless MAC Collision Avoidance) withStochastic Network Calculus (SNC) based strategy for QoS examination. To make a model, we develop a Stochastic Curve model for Gilbert-Elliot Channel and separate the traffic passing on limit concerning a given confirmation at the stream level.

To enhance the efficiency of traditional hand-shacking, the UWMACA protocol with Stochastic Network [16] Calculus is invented to reduce the collision rate and increase the successful communication between the underwater farming bubbles to base Sink [17]. The rest of the investigation article is figured out as follows. Fragment II shows the Analysis and working of UWMACA. Portion III explains the stochastic network calculus setup with obscuring channel. Section IV focuses on the simulation results of the proposed scheme of UWMACA. Section V concludes the investigation.

\section{ANALYSIS OF MACA AND WORKING OF UWMACA-WIRELESS PROTOCOLS}

A. Analysis of MACA-Wireless protocol

The wireless protocol MACA follows the R-C-D-A mechanism [R-RTS, C-CTS, D-Data, and A-ACK] to interchanges the data between the nodes. The RTS and CTS messages will help the node to avoid the collision occurrences between two intended devices. 


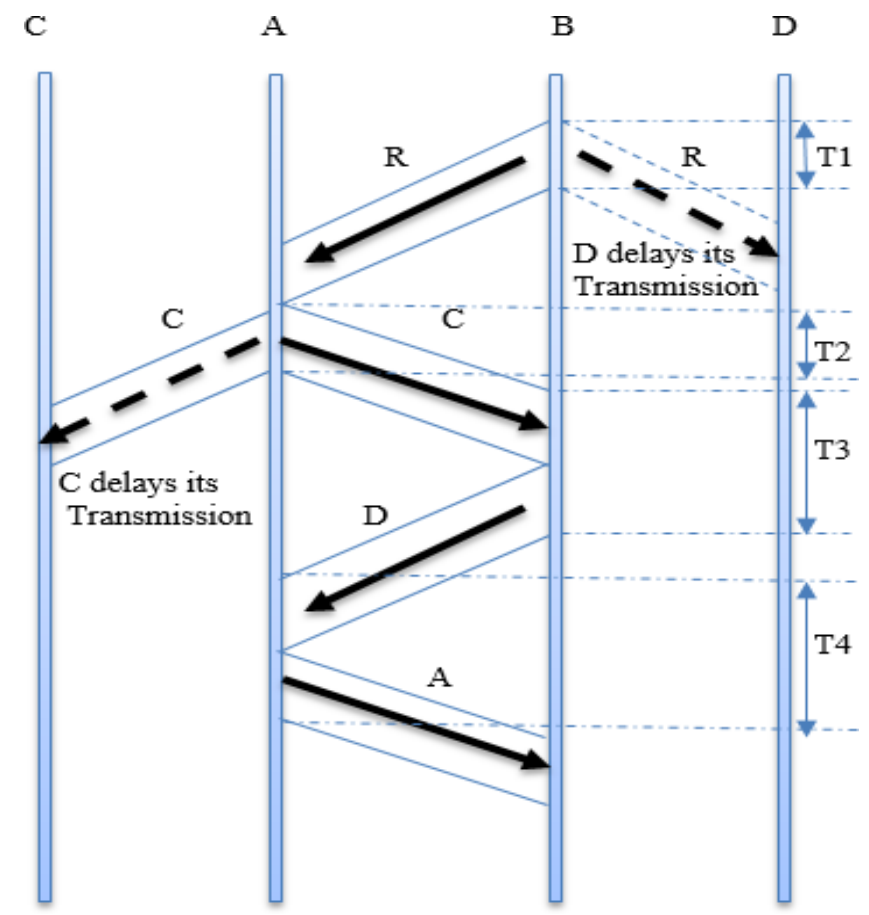

Figure 2. Information exchange using R-C-D-A in MACA-wireless protocol

Fig.2 illustrates a controlled data flow between nodes. Node B willing to communicate with node A. Node B exchanges RTS messages to node A. But the same RTScan view by nearest node D and understand the communication occurrences [19]. Node D will go to waiting mode until the reception of the communication termination message. Node A sends a CTS message when the node is free. The CTS message can be viewed by nearest node $\mathrm{C}$. node $\mathrm{C}$ will enter to waiting mode until the completion of communication between $\mathrm{A}$ and $\mathrm{B}$. once the CTS message is received from node A, the data exchange will happen. For every successful data exchange the ACK will be shared by node A.Underwater channel has the less busy time due to long propagation delay, which means that most of the time the channel will be idle [20]. Ti denotes the total amount of time for entire communication starts from RTS to ACK. The total communication time is expressed as,

$$
T i=\frac{P(\text { rts })+P(c t s)+P(\text { data })+P(\text { ack })}{R(\text { rate })}+\frac{4 * \mathbb{D}}{S}
$$

Where $\mathrm{P}(\mathrm{rts}), \mathrm{P}(\mathrm{cts}), \mathrm{P}($ data) and $\mathrm{P}$ (ack) denotes the packet size of the R-C-D-A mechanism. Di represents the Distance between the nodes, R(rate) denotes the data rate between nodes. Here for simulation, we have considered an equal rate for both nodes. $\mathrm{S}$ denotes the speed of the acoustic wave.The busy time Bt of the channel evaluates as,

$$
B_{t}=\frac{P(r t s)+P(c t s)+P(\text { data })+P(\text { ack })}{R(\text { rate })}
$$

The ratio of busy duration $\rho$ is denoted as,

$$
\rho=\frac{B t}{T i} * 100 \%
$$

The typical example for MACA - wireless protocol, let $\mathrm{P}(\mathrm{rts}), \mathrm{P}(\mathrm{cts}), \mathrm{P}($ data), and $\mathrm{P}$ (ack)hasan equal length of a packet. (Ex. 100 bits), $\mathrm{P}($ data $)=1024 \mathrm{~B}, \mathrm{Di}=2000$ meter, $\mathrm{R}($ rate $)=1000 \mathrm{~b} / \mathrm{s}$, Speed $\mathrm{S}=1500 \mathrm{~m} / \mathrm{s}$. substituting the values in (1), (2), and (3), it yields $T i \simeq 6692 \mathrm{~s}, \mathrm{~B}_{-} \mathrm{t}=1.3215 \mathrm{~s}, \rho \simeq 19.91 \%$. Hence $\mathrm{B}_{-} \mathrm{t}<\mathrm{Ti}$. It means that channel will be idle for the maximum amount of time. So MACA-wireless protocol is insufficient for the underwater environment. If the distance is increased between nodes. Another disadvantage is propagation delay will be less because it interleaved with the busy time. 


\section{B. Working principle of UWMACA-Wireless protocol}

MACA-wireless protocol yields $B_{-} \mathrm{t}<\mathrm{Ti}$. The channel utilization is very less and propagation delay interfered with the busy time. To overcome these issues, UMACA-wireless protocol giving much more attention to every packet that extracts the information of the sender, receiver, and busy state of neighbors during R-C-D-A.

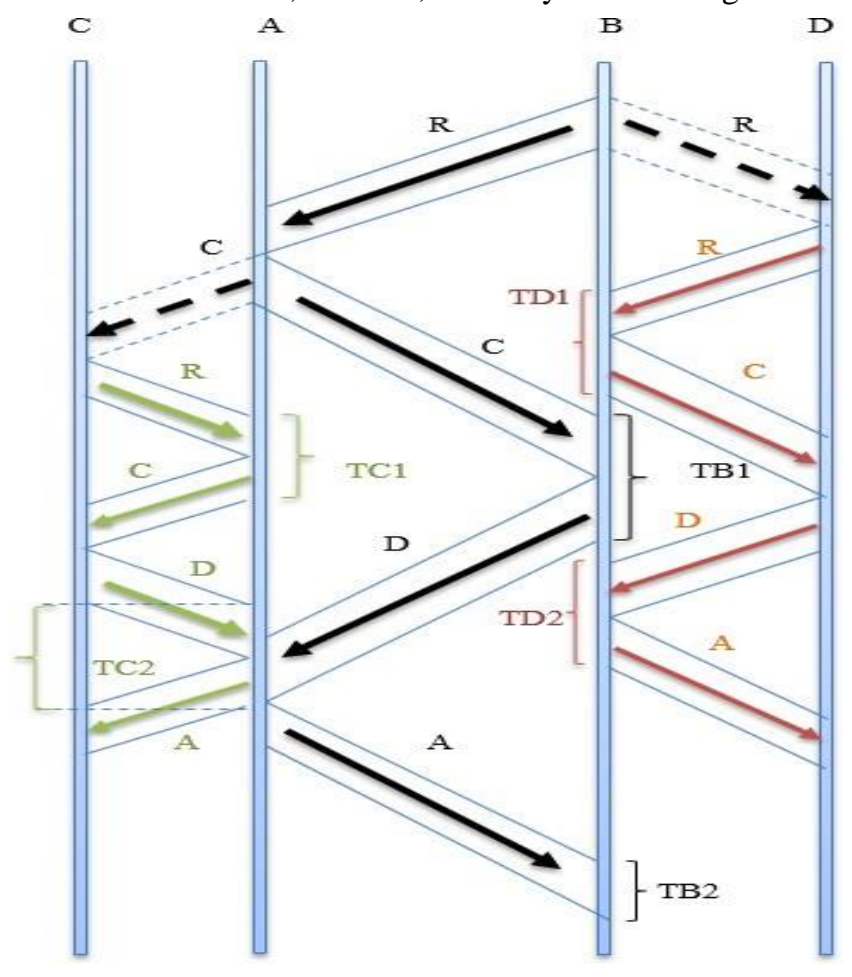

Figure 3. Information exchange using R-C-D-A in UWMACA-wireless protocol

According to the UMACA - Wireless protocol, every node shall listen to the connection and listen closely to every other package it gets to know, then collect details about both the nodes, as well as the active timeframes of strangers.UMACA - Wireless protocol utilize the channel's network latency time, allowing nodes to communicate with many other peers during the R-C-D-A exchange era. Crashes will never happen when active intervals of multiple nodes aren't overlapping at one another. Figure 3, illustrates the R-C-D-A messages in a three-way. $\mathrm{A}$ is conversing to $\mathrm{B}$, and $\mathrm{C}$ will send messages to $\mathrm{A}$ through inter-leaving their active intervals $\mathrm{TC}$ and TA mostly during communication time of A and B. Likewise, B will send information to A and D in the same exchange time provided the active intervals TD and TB do not overlap.

The active periods are split into 2 parts, TB1 \& TB2. TB1 is the time it takes from the start T1 and receiving the CTS to the final time T2 of transmitting data. TB2 has to be the time T3 required from the start of collecting ACK to the final time T4 of acquiring ACK.The transmission time can be computed as illustrated in Figure 3.

$$
\begin{aligned}
& T 1=T_{B C}+T_{r t s}+2 \partial B A \\
& T 2=T 1+T_{c t s}+T \text { data } \\
& \quad T B 1=[T 1, T 2] \\
& T 3=T_{B C}+T_{r t s}+T_{c t s}+T_{\text {data }}+4 \partial B A \\
& T 4=T 3+T_{A C K} \\
& T B 2=[T 3, T 4]
\end{aligned}
$$

Where,

$T_{B C}=$ Present time of the node B sending RTS

$T_{r t s}=\mathrm{RTS}, T_{c t s}=\mathrm{CTS}, T_{\text {data }}=\mathrm{DATA}, T_{A C K}=\mathrm{ACK}$

$\partial B A=$ Propagation delay between $A \& B$

During the busy time TB of node $B$ exchange the message to A, it can be written as,

$T B=T B 1 \bigcup T B 2(6)$

D, as A's neighbor, hears the RTS packet and is aware of the busy length TB. If D needs to send data to B, it must also calculate the busy durations TD1 and TD2, which are the output of a new message exchange between 
D and B.TD1 is the time between both the initial stage T5 of obtaining RTS D and the finish times T6 of transmitting CTS D, and TD2 is the time between both the initial stage T7 of obtaining DATA D as well as the end time T6 of transmitting CTS D.

$$
\begin{aligned}
& T 5=T_{D C}+\partial D B \\
& T 6=T 5+T_{r t s}+T_{c t s} \\
& T D 1=[T 5, T 6] \\
& T 7=T_{D C}+T_{r t s}+T_{c t s}+3 \partial D B \\
& T 8=T 7+T_{\text {data }}+T_{A C K} \\
& T D 2=[T 7, T 8] \\
& \text { Where, } \\
& T_{B C}=\text { Present time of the node D sending RTS } \\
& \partial D B=\text { Propagation delay between D\&B }
\end{aligned}
$$

During the busy time TD of node $\mathrm{D}$ exchange the message to $\mathrm{B}$, it can be written as,

$$
T D=T D 1 \bigcup T D 2
$$

\section{SNC -Notations for Channel utilization for UWMACA communication}

SNC has its root from the Queuing hypothesis [13]. A cycle time is characterized as the capacity of time $t$. The different components arrival rate is $\operatorname{Arrv}(\mathrm{t})$ (Arrival or appearance measure), the measure of (t) (departure takeoff measure), the Service is denoted as Serv ( $t$ ) (Service measure), and the measure of I (t) (Impairment measure).We expected all cycle are non-negative and expanding capacities and by conventiont $=0$,

$$
\text { i.e., } \operatorname{Arrv}(0)=\operatorname{Dept}(0)=\operatorname{Serv}(0)=\mathrm{I}(0)=0 \text {. }
$$

For any $0 \leq \mathrm{s}$ erv $\leq \mathrm{t}$,

Default values are, $\operatorname{Arrv}(0)=\operatorname{Dept}(0)=\operatorname{Serv}(0)=0$.

The non-negative wide detecting expanding capacity is indicating as $\mathrm{f}$ the arrangement of non-negative widedetecting expanding capacities, and $\mathrm{f}^{-}$the arrangement of non-negative diminishing capacities,

$$
\begin{aligned}
& \mathrm{f}=\{\mathrm{f} \dashv \operatorname{un}(.): \forall 0 \leq \mathrm{x} 1 \leq \mathrm{y} 1,0 \leq \mathrm{fun}(\mathrm{x} 1) \leq \mathrm{fun}(\mathrm{y} 1 \vdash)\} \\
& \mathrm{f}=\{\operatorname{fun}(.): \forall 0 \leq \mathrm{x} 1 \leq \mathrm{y} 1,0 \leq \mathrm{fun}(\mathrm{y} 1) \leq \mathrm{fun}(\mathrm{x} 1)\}
\end{aligned}
$$

Variable with randomness is denoted asC1, its functional distribution is denoted byFun_c $(C) \equiv \operatorname{Prob}\{C \leq c\}$, fitsf, and HDF- Harmonizing distribution function, f_c $(C) \equiv \operatorname{Prob}\{C>c\}$, fits to $f$. For modeling, the bounding function needs a stronger requirement on the execution by Ĝroles in $\mathrm{f}$ Where $\mathrm{g}(.) \in \hat{\mathrm{G}}, \mathrm{x} 1 \geq 0$ and $\hat{\mathrm{G}}$ for $\mathrm{n} 1 \geq 0$, i.e.,

$$
\hat{\mathrm{G}}=\left\{\mathrm{g}(.): \forall \mathrm{n} 1 \geq 0,\left(\int_{x 1}^{\infty} \mathrm{dy} 1\right) \quad \mathrm{g}(\mathrm{y} 1) \in \hat{\mathrm{G}}\right\}
$$

\section{SIMULATION AND PERFORMANCE BOUNDS}

The main intention of our work is to simulate the UWMACA-W and MACA-W protocols to investigate the throughput and delays. The node is deployed in every underwater bubble. Similarly, every bubble node can exchange the data and exchange the data to the base station (like mesh topology).

The communication distance used for simulation is $1000 \mathrm{~m}, 1500 \mathrm{~m}$, and 2000 meters between nodes and base station. The simulation work was done at the reverbed simulator. All nodes can make half-duplex and receiving signals from or transmitting in all directions with each other. The fixed transmission rate is $1000 \mathrm{~b} / \mathrm{s}$. The other simulation setup parameters are discussed in Table 1.

TABLE I

ATTRIBUTES FOR SIMULATION

\begin{tabular}{|l|l|}
\hline Topology & Mesh Topology \\
\hline Simulator & Reverbed \\
\hline Communication mode & Half-duplex \\
\hline Direction & Omnidirectional \\
\hline Deployed node Count & 14 \\
\hline Adjournment (delay) & $4 \mathrm{~s}$ \\
\hline Speed & $1500 \mathrm{~m} / \mathrm{s}$ \\
\hline
\end{tabular}




\begin{tabular}{|l|l|}
\hline Distribution & Poisson \\
\hline $\begin{array}{l}\text { Distance of } \\
\text { communication }\end{array}$ & $1000 \mathrm{~m}-2000 \mathrm{~m}$ \\
\hline Data size & $1000 \mathrm{~b} / \mathrm{s}$ \\
\hline $\begin{array}{l}\text { Delay bound and } \\
\text { Backlog }\end{array}$ & $\mathrm{SNC}$ \\
\hline Rts, Cts,Ack Size & $100 \mathrm{~b} / \mathrm{s}$ \\
\hline $\begin{array}{l}\text { Buffer size of every } \\
\text { node }\end{array}$ & 5 packet size \\
\hline Delay violation prob. & $0.0-1.0$ \\
\hline
\end{tabular}

A simulation arrangement for investigating the MAC layer with fading effects inan underwater acoustic network is deployed using nodes along with requirements. Fig. 4 shows the simulation node setup in the reverbed simulation atmosphere with fourteen nodes. The two halfway hand-off hubs screen the information appearance rate and the administration rate among the hubs. Reverbed is a medium that a matter of course strengthens remote acoustic signal correspondence. For each pair of communication and channels, the remote transmission preparation can be portrayed by a progrescsion of sub-transmission blocks.

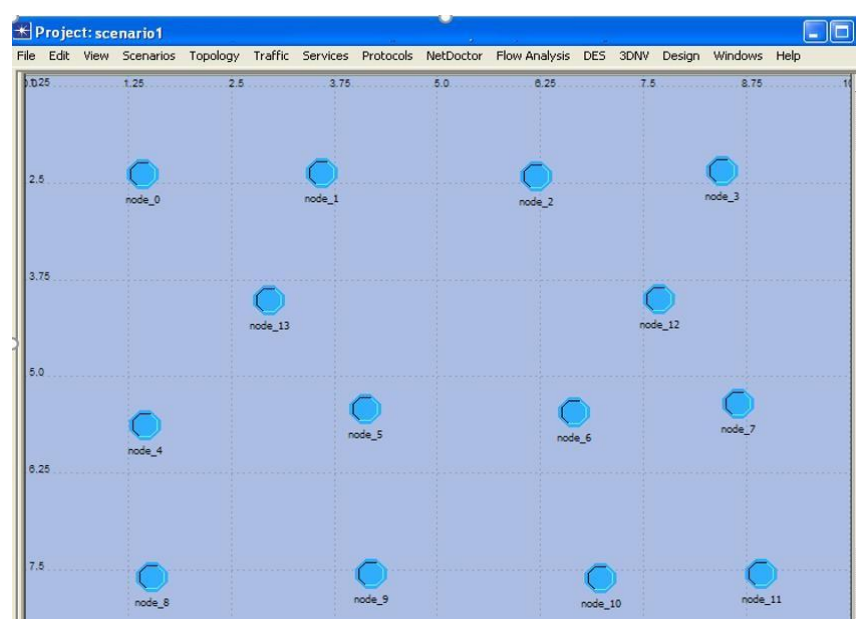

Figure 4. Delay Guarantee vs Throughput

The Figure 5. Shows the performance of UWMACA-Wireless protocol is better than MACA-Wireless Protocol. Here UWMACA-W achieved better throughput than the MACA-W. The main advantage of UWMACA-W is utilizing the ide time due to the propagation delay. Figure 6. Describe the average delay variations between UWMACA-Wireless protocol and MACA-Wireless Protocol. Here UWMACA-Wireless protocol has better performances than the MACA-Wireless protocol because the UWMACA-W utilizing the propagation delay use for other control information transaction between nodes. The smaller payload is minimally caused by the delay, the large payload handled buffer in each node.

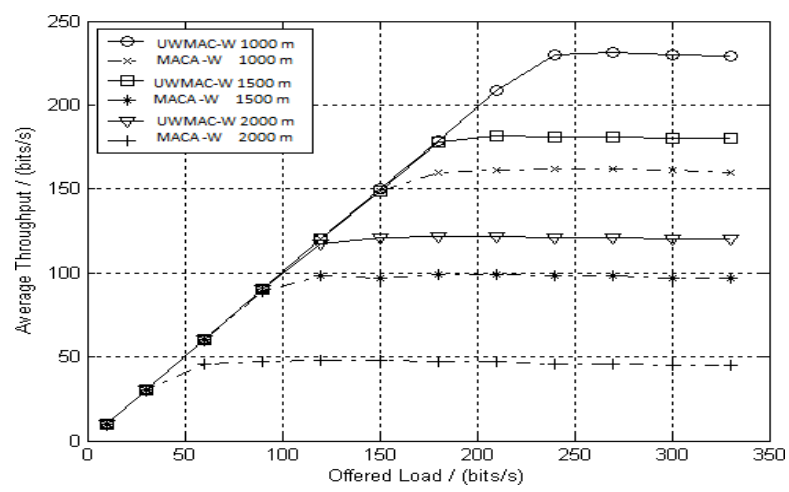

Figure 5. Average throughput e vs Load 


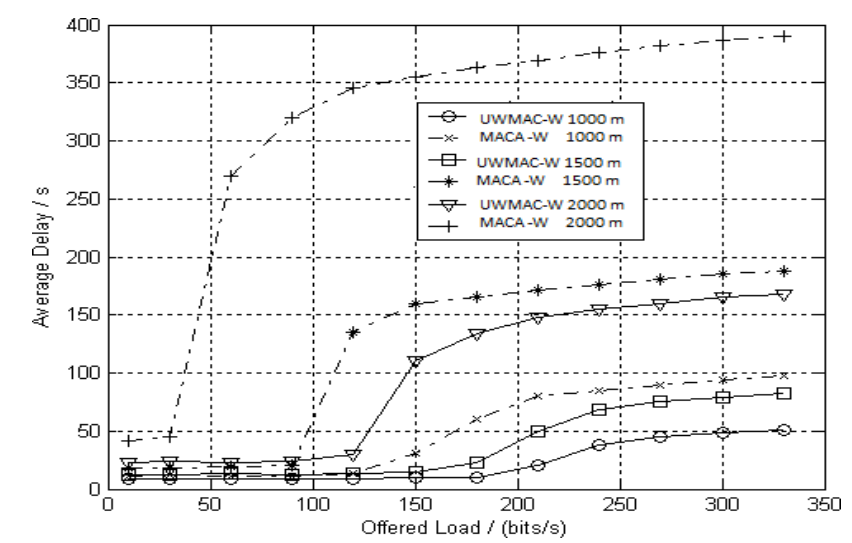

Figure 6. Average Delay vs Load

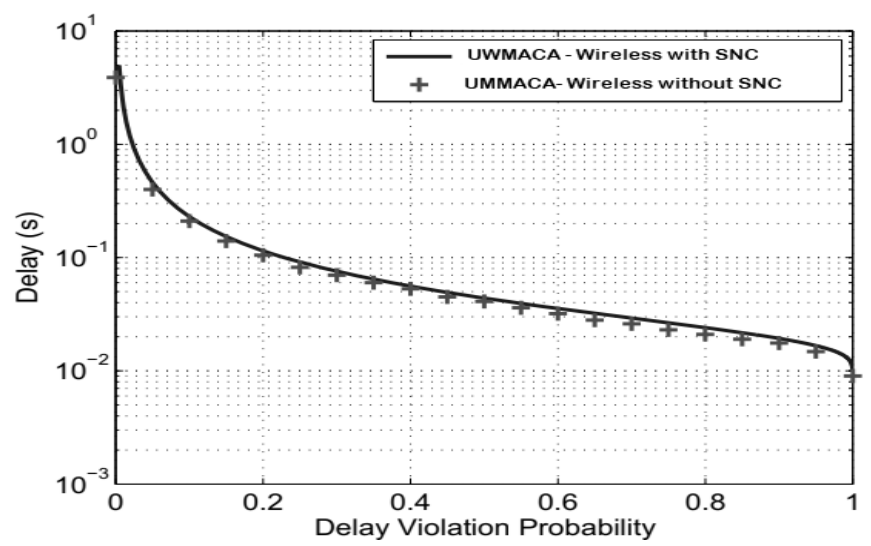

Figure 7. UWMCA-W Delay Vs Delay Violation Probability

Figure 7. Describes the delays in a simulation, where Source sent 1000 packets. From this, the packet deferrals are very dissimilar, unpredictable from $0.01 \mathrm{~s}$ to $5 \mathrm{~s}$. Few packets experience high delays due to the distance and delay violation probability. From these simulation results, we can derive the delay bounds with corresponding violation probabilities. UWMACA-W simulated with and without SNC, the deferral rates are $5.19 \%(1000 \mathrm{~m}), 9.88 \%(1500 \mathrm{~m})$, and $13.5 \%$ (2000 m), respectively.

\section{CONCLUSION}

In this exploration work, we have done simulation work for monitoring the growth of the underwater agriculture forming. For communication purposes, we have presented random access and delay-tolerant MAC protocol (UWMACA-Wireless) to adapt to the ocean environment and avoid collision occurrences. Channel allocation and data transmission occurring with blurring impacts of the acoustic channel utilizing Stochastic Network Calculus. The variation with and without SNC shows the deferral rate between dealy concerning delay violation probability. The control messages can occur between the devices when the propagation delay occurs during the R-C-D-A. Furthermore, message transfers based upon the neighbor's active timeframes limit the issue of concealed and uncovered nodes.

\section{References}

1. Jakobsson, Martin, Ron Macnab, Larry Mayer, Robert Anderson, Margo Edwards, Jörn Hatzky, Hans Werner Schenke, and Paul Johnson. "An improved bathymetric portrayal of the Arctic Ocean: Implications for ocean modeling and geological, geophysical oceanographic analyses", Geophysical Research Letters, Vol.35, No. 7, 2008

2. Ian F. Akyildiz, Dario Pompili, Tommaso Melodia, "Underwater acoustic sensor networks: research challenges", Elsevier - Ad Hoc Networks, Vol. 3, No.3, pp.257-279, 2005

3. Christhu Raj M R, Rajeev Sukumaran, "Modeling UWSN Simulators- A Taxonomy", International Journal of Computer, Electrical, Automation, Control, and Information Engineering, World Academy of Science, Engineering and Technology, Vol. 9, No. 2, 2015, pp. 585-592.

4. Christhu Raj M R, Rajeev Sukumaran, "Modeling and Simulation of Acoustic Link Using Mackenize Propagation Speed Equation", International Journal of Computer, Electrical, Automation, Control, and 
Information Engineering, World Academy of Science, Engineering and Technology, Vol. 9, No. 10, 2015, pp. 1874-1882.

5. Christhu Raj M R, Rajeev Sukumaran, "Stochastic Network Calculus Model for AWGN Fading in Underwater Wireless Communication Networks", Australian Journal of Basic and Applied Sciences, 9(31), 2015, pp. 253-262.

6. Wan Du, Fabien Mieyeville1, David Navarro, Ian O'Connor,Laurent Carrel1, "Modeling and simulation of networked low-power embedded systems: a taxonomy", EURASIP Journal on Wireless Communications and Networking, Vol.2014, No.1, pp. 1-12, 2014

7. Saravanan M., Rajeev Sukumaran., Christhuraj M. R., Manikandan T. T., "SNC for Modeling Delay Dissimilarity in Acoustic Mode Communication for Underwater Wireless Multichannel Communication", IJAST, vol. 29, no. 3, pp. 13625 - 13634, Mar. 2020.

8. M. Saravanan, R. Sukumaran, M. R. Christhuraj and T. T. Manikandan, "Delay in underwater Acoustic Wireless single channel communication: Stochastic Network Calculus," 2020 Fourth International Conference on I-SMAC (IoT in Social, Mobile, Analytics and Cloud) (I-SMAC), Palladam, India, 2020, pp. 1138-1144, doi: 10.1109/I-SMAC49090.2020.9243399.

9. M. Saravanan, R. Sukumaran, M. R. Christhuraj and T. T. Manikandan, "Delay analysis of medium access control layer in underwater acoustic wireless communication: Stochastic network calculus", Journal of Green EngineeringVolume 10, Issue 12, December 2020, Pages 13247-13262

10. Jens B. Schmitt, U.Roedig, "Sensor network Calculus- a framework for worst-case analysis". IEEE/ACM International Conference on Distributed Computing in Sensor Systems(DCOSS'05), 2005.

11. Y. Xue, B. Ramamurthy, and M. C. Vuran "SDRCS: A service-differentiated real-time communication scheme for event sensing in wireless sensor networks", Computer. Networks., vol. 55, pp.3287 -3302, 2011

12. S. Hariharan and N. B. Shroff: "Maximizing Aggregated Information in Sensor Networks under Deadline Constraints, IEEE Transactions. on Automatic Control (Special Issue on Wireless Sensor and Actuator Networks), 56(10), pages 2369-2380, 2011

13. Kashif Mahmood, Mikko Vehkapera, Yuming Jang, "Delay Constrained Throughput Analysis of SISO”. `The 3rd IEEE Conference on Network Infrastructure and Digital Content (IC-NIDC), 2012.

14. M. Saravanan, R. Sukumaran, M. R. Christhuraj and T. T. Manikandan, "Survey of Various Mathematical Approaches suitable for Underwater Wireless Communication," 2020 4th International Conference on Intelligent Computing and Control Systems (ICICCS), Madurai, India, 2020, pp. 560565, doi: 10.1109/ICICCS48265.2020.9120995.

15. M. Fidler, "A network calculus approach to probabilistic quality of service analysis of fading channels," In Proceedings. of GLOBECOM, 2006.

16. K. Mahmood, M. Vehkapera, and Y. Jiang, "Delay constrained throughput analysis of a correlated MIMO wireless channel," IEEE International Conference on Computer Communications and Networks (ICCCN), August 2011.

17. K. Mahmood, A. Rizk, and Y. Jiang, "On the flow-level delay of a spatial multiplexing MIMO wireless channel," IEEE International Conference on Communications (ICC), June 2011.

18. K. Mahmood, M. Vehkapera, and Y. Jiang, "Performance of multiuser CDMA receivers with bursty traffic and delay constraints," IEEE International Conference on Computing, Networking and Communications (ICNC), 2012.

19. Huimin She, Zhonghai Lu, Axel Jantsch, Dian Zhou, Li-Rong Zhe "Modeling and Analysis of Rayleigh Fading Channels using Stochastic Network Calculus", IEEE Conference on WCNC, pp. 1056-1061, 2011

20. M. Fidler," A end to end probabilistic network calculus with Moment Generating Functions", In proceedings of the 14th IEEE International Workshop on Quality of Service(IWQoS), pp. 261-270, 2006. 